\title{
Cold PSM, but not TRAIL, triggers autophagic cell death: A therapeutic advantage of PSM over TRAIL
}

\author{
TOMOHISA ITO $^{1}$, TAKASHI ANDO ${ }^{2}$, MIKI SUZUKI-KARASAKI ${ }^{1,3}$, TOMOHIKO TOKUNAGA $^{4}$, \\ YUKIHIRO YOSHIDA ${ }^{1}$, TOYOKO OCHIAI ${ }^{5}$, YASUAKI TOKUHASHI ${ }^{1}$ and YOSHIHIRO SUZUKI-KARASAKI ${ }^{3,6}$ \\ ${ }^{1}$ Department of Orthopedic Surgery, Nihon University School of Medicine, Tokyo 173-8610; \\ ${ }^{2}$ Department of Orthopedic Surgery, Yamanashi University School of Medicine, Yamanashi, Chuo, Yamanashi 409-3898; \\ ${ }^{3}$ Plasma ChemiBio Laboratory, Nasushiobara, Tochigi 329-2813; ${ }^{4}$ Division of General Medicine, \\ Nihon University School of Medicine, Tokyo 173-8610; ${ }^{5}$ Department of Dermatology, Nihon University Hospital, \\ Tokyo 101-8309; ${ }^{6}$ Division of Physiology, Department of Biomedical Sciences, \\ Nihon University School of Medicine, Tokyo 173-8610, Japan
}

Received November 2, 2017; Accepted March 26, 2018

DOI: 10.3892/ijo.2018.4413

\begin{abstract}
Tumor necrosis factor-related apoptosis-inducing ligand (TRAIL) and cold plasma-stimulated medium (PSM) are promising novel anticancer tools due to their strong anticancer activities and high tumor-selectivity. The present study demonstrated that PSM and TRAIL may trigger autophagy in human malignant melanoma and osteosarcoma cells. Live-cell imaging revealed that even under nutritional and stress-free conditions, these cells possessed a substantial level of autophagosomes, which were localized in the cytoplasm separately from tubular mitochondria. In response to cytotoxic levels of PSM, the mitochondria became highly fragmented, and aggregated and colocalized with the autophagosomes. The cytotoxic effects of PSM were suppressed in response to various pharmacological autophagy inhibitors, including 3-methyladenine (3-MA) and bafilomycin A1, thus indicating the induction of autophagic cell death (ACD). Lethal levels of PSM also resulted in non-apoptotic, non-autophagic cell death in a reactive oxygen species-dependent manner under certain circumstances. Furthermore, TRAIL exhibited only a modest cytotoxicity toward these tumor cells, and did not induce ACD and mitochondrial aberration. The combined use of TRAIL and subtoxic concentrations of 3-MA resulted in decreased basal autophagy, increased mitochondrial aberration, colocalization with autophagosomes and apoptosis. These results indicated that PSM may induce ACD, whereas TRAIL may
\end{abstract}

Correspondence to: Dr Yoshihiro Suzuki-Karasaki, Plasma ChemiBio Laboratory, Takaatsu 398-4, Nasushiobara, Tochigi 329-2813, Japan E-mail: suzuki.pcbl@gmail.com

Key words: cold plasma-stimulated medium, tumor necrosis factorrelated apoptosis-inducing ligand, autophagy, autophagic cell death, mitophagy trigger cytoprotective autophagy that compromises apoptosis. To the best of our knowledge, the present study is the first to demonstrate that PSM can induce ACD in human cancer cells. These findings provide a rationale for the advantage of PSM over TRAIL in the destruction of apoptosis-resistant melanoma and osteosarcoma cells.

\section{Introduction}

Tumor necrosis factor (TNF)-related apoptosis-inducing ligand (TRAIL) is a member of the TNF superfamily, which preferentially kills malignant cells over nontransformed cells (1-4). TRAIL can induce extrinsic and intrinsic death pathways by binding its specific receptors with death domain TRAIL receptor (TRAIL-R)1/death receptor (DR)4 and TRAIL-R2/DR5 $(5,6)$. However, some cancer cell types are inherently resistant to TRAIL, despite expressing deathinducing receptors (7-11). Furthermore, some cell types acquire considerable tolerance to TRAIL during prolonged treatment. Accordingly, current clinical trials have been disappointing, and the combined use of agents that overcome drug resistance is necessary for efficient TRAIL therapy. Non-thermal (cold) atmospheric plasma (CAP) has emerged as another promising means of cancer treatment, since like TRAIL, it kills various cancer cells while sparing nontransformed cells under optimal conditions (12-15). Cold plasma-stimulated medium (PSM) also exhibits vigorous and tumor-selective anticancer activities (16-19) and has emerged as an alternative method of direct CAP irradiation; PSM is better than direct CAP irradiation for systematic or local administration to deep tissues.

Cancer cells, including malignant melanoma (MM) and osteosarcoma (OS) cells, are characterized by their intrinsic resistance to apoptosis; in addition, they frequently become more tolerant to numerous apoptosis-inducing antitumor drugs. Nevertheless, the majority of conventional drugs primarily kill cells by apoptosis. Accordingly, current chemotherapy toward these cancers is severely compromised by intrinsic and acquired resistance; therefore, induction of another mode 
of cell death may be a useful approach for the treatment of apoptosis-resistant cells $(20,21)$. Autophagy is a primary catabolic process that degrades cellular components and damaged organelles via lysosomes; this process copes with cellular stressors, such as starvation, and supplies energy and metabolic precursors. Autophagy consists of numerous processes, including induction of cytoplasmic double-layered membranes, which are known as phagophores, phagophore elongation and autophagosome formation, a fusion of autophagosomes with lysosomes, and degradation and recycling. All processes, from the formation of autophagosomes to the degradation of cellular components, are strictly regulated by autophagy-related (Atg) proteins that are encoded by Atg genes (22). Autophagy is classified into three different types: Macroautophagy (subsequently referred to as autophagy), microautophagy and chaperone-mediated autophagy. Autophagy is negatively regulated by mammalian target of rapamycin complex I in response to insulin and amino acid signals, and is driven transiently via removal of its suppression through the depletion of these nutrients (23-25). Therefore, autophagy is of particular importance for the survival of constitutively proliferating cells, such as cancer cells, that are regularly imposed to energy demands $(20,26)$. In addition, autophagy contributes to cancer cell survival by removing damaged organelles, including mitochondria and endoplasmic reticulum (ER) by microautophagy, which is also known as mitophagy and ERphagy, respectively. These damaged organelles are degraded via lysosomal enzymes following engulfment into autophagosomes; such quality control is crucial for cell survival. Conversely, autophagy is also characterized by a unique cell death pathway that acts as a tumor suppressor when it leads to autophagic cell death (ACD) (27-29).

Our previous study revealed that PSM prepared by CAP irradiation of Dulbecco's modified Eagle's medium (DMEM) could kill an array of MM, OS and lung cancer cells, while sparing nontransformed melanocytes and fibroblasts (30). In addition, PSM led to increased caspase-3/7 activity, and modest cleavage of caspase- 9 , caspase-3/7 and poly ADP-ribose polymerase; furthermore, caspase-3/7-specific inhibitors failed to suppress cell death. Therefore, the present study aimed to examine the possibility that PSM may induce another cell death modality. The results demonstrated that PSM can trigger ACD in MM and OS cells.

\section{Materials and methods}

Materials. Soluble recombinant human TRAIL was obtained from Enzo Life Sciences, Inc. (Farmingdale, NY, USA). 3-Methyladenine (3-MA), chloroquine (CQ) and bafilomycin A1 (Baf) were obtained from Sigma-Aldrich (Merck KGaA, Darmstadt, Germany). The pan-caspaseinhibitor z-VAD-fluorometheylketone (Z-VAD-FMK) was purchased from Merck Ltd. (Tokyo, Japan). All insoluble reagents were dissolved in dimethyl sulfoxide (DMSO) and diluted with high glucose-containing DMEM supplemented with $10 \%$ fetal bovine serum (FBS) (both from Sigma-Aldrich; Merck KGaA) or Hank's balanced salt solution (HBSS; pH 7.4; Nissui Pharmaceutical Co., Ltd., Tokyo, Japan) (final DMSO concentration, $<0.1 \%$ ) prior to use. The manganese-porphyrin superoxide dismutase mimetic MnTBaP (Enzo Life Sciences,
Inc.) was dissolved in $1 \mathrm{mM} \mathrm{NaOH}(\mathrm{pH} \mathrm{8.0)}$ and HBSS was added to lower the $\mathrm{pH}$ to 7.4 .

Cell culture. Human A375 MM cells [American Type Culture Collection (ATCC) ${ }^{\circledR}$ cell number CRL-1619) were obtained from ATCC (Manassas, VA, USA). A2058 MM cells (cell number IFO 50276) and human A549 lung adenocarcinoma cells (cell number JCRB0076) were purchased from the JCRB Cell Bank of National Institutes of Biomedical Innovation, Health, and Nutrition (Osaka, Japan). Human HOS (TE85) OS cells (cell number RCB0992), SAOS-2 OS cells (cell number RCB0428) and 143B cells (cell number RCB0701) were obtained from the Riken BioResource Center (Tsukuba, Japan). Human dermal fibroblast (HDF) cells from facial dermis were obtained from Cell Applications (San Diego, CA, USA). All cells were cultured in $10 \%$ FBS/DMEM supplemented with $100 \mathrm{U} / \mathrm{ml}$ penicillin and $100 \mu \mathrm{g}$ streptomycin (Thermo Fisher Scientific, Inc., Waltham, MA, USA) in a humidified atmosphere containing $95 \%$ air $/ 5 \% \mathrm{CO}_{2}$ at $37^{\circ} \mathrm{C}$. The cells were harvested by incubating with $0.25 \%$ trypsin-EDTA (Thermo Fisher Scientific, Inc.) for $5 \mathrm{~min}$ at $37^{\circ} \mathrm{C}$. Throughout the study, various cell lines were used in each experiment to determine whether the reactions observed were specific for the cell line used or were general among the various cell lines. A2058 and HOS (or SAOS-2) cells were used as the main representatives of MM and OS cells, respectively. In some experiments, autophagy was compared between normally grown A375 (confluence, $70-80 \%$ ) cells and overgrown (confluent, partially floating) cells.

PSM preparation. CAP was generated using an originallydeveloped low-frequency plasma jet device equipped with an asymmetrical dielectric barrier discharge, as previously described (30). The typical frequency was $20 \mathrm{kHz}$, with a peak voltage of $8 \mathrm{kV}$, a current of $20 \mathrm{~mA}$ and a helium flow rate of $300 \mathrm{ml} / \mathrm{min}$. PSM was prepared by irradiating $1 \mathrm{ml}$ FBS/DMEM with CAP for 5 min once. The original PSM $(100 \%)$ was diluted to a final concentration of $25 \%$ with FBS/DMEM (for cell experiments) or HBSS (for biochemical experiments), and indicated as PSM (25\%).

Cell viability assay. Cell viability was measured according to the WST-8 assay using Cell Counting Reagent SF (Nacalai Tesque, Inc., Kyoto, Japan) as previously described (31). This is a colorimetric assay that detects viability based on the formation of a water-soluble formazan product. Briefly, the cells were seeded at a density of $8 \times 10^{3}$ cells/well in 96 -well plates (Corning Incorporated, Corning, NY, USA) and cultured with PSM (12.5, 25, 50, and 100\%), $100 \mathrm{ng} / \mathrm{ml}$ TRAIL, 3-MA (1.3-5 mM), CQ (100 and $300 \mu \mathrm{M})$, and Baf (100 and $300 \mathrm{nM})$ alone or in combination for $72 \mathrm{~h}$ at $37^{\circ} \mathrm{C}$, prior to the addition of $10 \mu \mathrm{l}$ cell counting reagent $\mathrm{SF}$ for $1 \mathrm{~h}$. For some experiments, the cells were cultured with the aforementioned reagents in the presence of $10 \mu \mathrm{M} Z$-VAD-FMK or $30 \mu \mathrm{M}$ MnTBaP. Absorbance was measured at $450 \mathrm{~nm}$ using an ARVO MX microplate reader (PerkinElmer Japan Co., Ltd., Yokohama, Japan).

Live-cell imaging. The mitochondrial network was analyzed as previously described (32) with minor modifications. Briefly, 
cells in FBS/DMEM ( $3 \times 10^{4} /$ well) adherent on an 8-well chambered coverslip with a glass bottom (Imaging Chamber $8 \mathrm{CG}$; Zell-Kontakt GmbH, Nörten-Hardenberg, Germany) were treated with PSM (25 and 100\%), $100 \mathrm{ng} / \mathrm{ml}$ TRAIL, 0.5 or $1 \mu \mathrm{M}$ rapamycin and $5 \mathrm{mM} 3-\mathrm{MA}$ alone or in combination for $24 \mathrm{~h}$ in a humidified atmosphere containing $95 \%$ air $/ 5 \% \mathrm{CO}_{2}$ at $37^{\circ} \mathrm{C}$. Following media removal by aspiration, the cells were washed with fresh FBS/DMEM and stained with $20 \mathrm{nM}$ MitoTracker Red CMXRos for $1 \mathrm{~h}$ at $37^{\circ} \mathrm{C}$ in the dark. The nuclei were counterstained with Hoechst 33342 . The cells were then washed with and immersed in FluoroBrite ${ }^{\mathrm{TM}}$ DMEM (Thermo Fisher Scientific, Inc.). Images were obtained using a BZ X-700 Fluorescence Microscope (Keyence Corporation, Osaka, Japan) equipped with a 100X, 1.40 n.a. UPLSAPO super-apochromat, coverslip-corrected oil objective (Olympus Corporation, Tokyo, Japan). Images were analyzed using BZ-H3A application software (Keyence Corporation) and National Institutes of Health (NIH) ImageJ software (bundled with 64-bit Java 1.8.0_112; NIH, Bethesda, MD, USA). The formation of autophagosomes was analyzed using the CYTO-ID ${ }^{\circledR}$ Autophagy Detection kit (Enzo Life Sciences, Inc.) according to the manufacturer's protocol. Briefly, cells were stained with CYTO-ID for $1 \mathrm{~h}$ at $37^{\circ} \mathrm{C}$ in the dark and treated with the aforementioned agents. For monitoring colocalization of mitochondria and autophagosomes, cells were coincidently stained with MitoTracker Red CMXRos and the CYTO-ID. Images were obtained using EVOS FL Cell Imaging system (Thermo Fisher Scientific, Inc.) equipped with a 40X, 0.60 n.a. LUCPLFLN objective (Olympus Corporation) as previously described (33).

Western blotting. Following treatment with PSM (25 and $100 \%$ ) for $12,24,36,48$ and $72 \mathrm{~h}$, cells were washed with $\mathrm{Ca}^{2+}-\mathrm{Mg}^{2+}$-free $\mathrm{PBS}$, and were lysed during $30 \mathrm{~min}$ agitation with CelLytic ${ }^{\mathrm{TM}}$ lysis buffer (Sigma-Aldrich; Merck KGaA) containing a protease inhibitor cocktail and a phosphatase inhibitor cocktail (both from Sigma-Aldrich; Merck KGaA). Cell debris was removed by centrifugation at 20,000 x g for $15 \mathrm{~min}$ at $4^{\circ} \mathrm{C}$. The supernatants were collected and protein concentrations were analyzed using a bicinchoninic acid protein assay (Thermo Fisher Scientific, Inc.), according to the manufacturer's protocol. Equal amounts of protein $(10 \mu \mathrm{g})$ were separated by $4-12 \%$ NuPage Bis-Tris acrylamide gels (Invitrogen; Thermo Fisher Scientific, Inc.) and were transferred to polyvinylidene difluoride membranes (Immobilon-P; EMD Millipore, Bedford, MA, USA). Blots were blocked for $30 \mathrm{~min}$ in Tris-buffered saline with $0.05 \%$ Tween-20 (TBST; Sigma-Aldrich; Merck KGaA) containing 2\% nonfat dry milk. The blots were then incubated with primary antibodies against p62 (PM045, 1:1,000; Medical \& Biological Laboratories Co., Ltd., Nagoya, Japan), microtubule-associated protein 1A/1B-light chain 3 (LC3; \#12741, 1:1,000) and GAPDH (\#5174, 1:1,000; Cell Signaling Technology, Inc., Danvers, MA, USA) overnight at $4^{\circ} \mathrm{C}$ in TBST containing $2 \%$ nonfat dry milk. After washing two times with TBST, membranes were incubated with horseradish peroxidaseconjugated goat anti-rabbit (\#7074, 1:2,000; Cell Signaling Technology, Inc.) for $1 \mathrm{~h}$ at room temperature. Subsequently, blots were washed three times with TBST and immersed in enhanced chemiluminescence reagent (GE Healthcare,
Chicago, IL, USA) to enhance the signals. The signals were then captured using an LAS-4000 Camera system (Fujifilm Corporation, Tokyo, Japan).

Statistical analysis. Data are presented as the means \pm standard deviation. Data were analyzed by one-way analysis of variance followed by Tukey's post hoc test using add-in software for Excel 2016 for Windows (Microsoft Corporation, Redmond, WA, USA). $\mathrm{P}<0.05$ was considered to indicate a statistically significant difference.

\section{Results}

Human MM and OS cells exhibit substantial basal autophagy. To gain insight into the possible role of autophagy in the antitumor activity of PSM, the present study evaluated its ability to modulate autophagic flux in MM and OS cells. CYTO-ID ${ }^{\circledR}$ Green is a cationic amphiphilic dye that can precisely monitor autophagic vacuoles without transfection. Live-cell imaging revealed a considerable number of CYTO-ID puncta in A375 and HOS cells in DMEM supplemented with $10 \%$ FBS. The CYTO-ID puncta markedly increased in response to $1 \mu \mathrm{M}$ rapamycin, whereas these puncta were decreased almost entirely following treatment with $5 \mathrm{mM} \mathrm{3-MA} \mathrm{(Fig.} \mathrm{1),} \mathrm{thus}$ validating that staining represents autophagosomes. Consistent with our previous study (30), treatment with PSM for $24 \mathrm{~h}$ resulted in robust cell damage. Accordingly, adherent spindle HOS and A549 cells became round, and some cells lost their adherence and integrity (Fig. 2A and B). Concomitantly, CYTO-ID puncta became clustered in these cells. Conversely, HDF cells possessed only modest basal CYTO-ID puncta and minimal changes in CYTO-ID puncta were observed following PSM treatment (Fig. 2C).

PSM induces colocalization of aggregated mitochondria and autophagosomes. Damaged mitochondria are removed by autophagic processes known as mitophagy. Accordingly, mitophagy controls the quality of mitochondria, thereby facilitating cell survival, whereas the excessive removal of mitochondria facilitates cell death $(34,35)$. Our previous study reported that PSM could induce excessive mitochondrial fragmentation and aggregation, alongside intensive mitochondrial damage (30). Therefore, the present study simultaneously monitored CYTO-ID puncta and mitochondrial morphology in live cells. Unstimulated A375 cells possessed tubular mitochondria and the CYTO-ID puncta that primarily diffused in the cytoplasm. In this case, the puncta and the mitochondria were separately located, as indicated by the minimal overlapping of green and red signals (Fig. 3A, upper panels). Following PSM treatment, the mitochondria became fragmented, punctate and aggregated, as previously described (30). Furthermore, some of the damaged round cells possessed clustered CYTO-ID puncta, the majority of which was colocalized with the aggregated mitochondria (Fig. 3A, lower panels). Even in the absence of insult, overgrown A375 cells became round and damaged (Fig. 3B, upper panels). Similarly, in PSM-treated cell images, damaged cells possessed numerous clustered CYTO-ID puncta, which were colocalized with aggregated mitochondria. To determine whether these effects were specific for PSM, the effects of TRAIL were determined. 
A

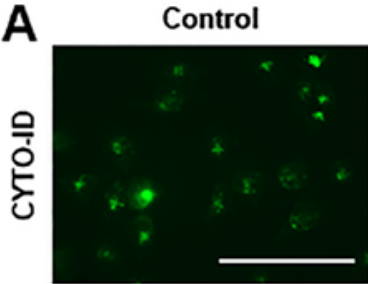

A375
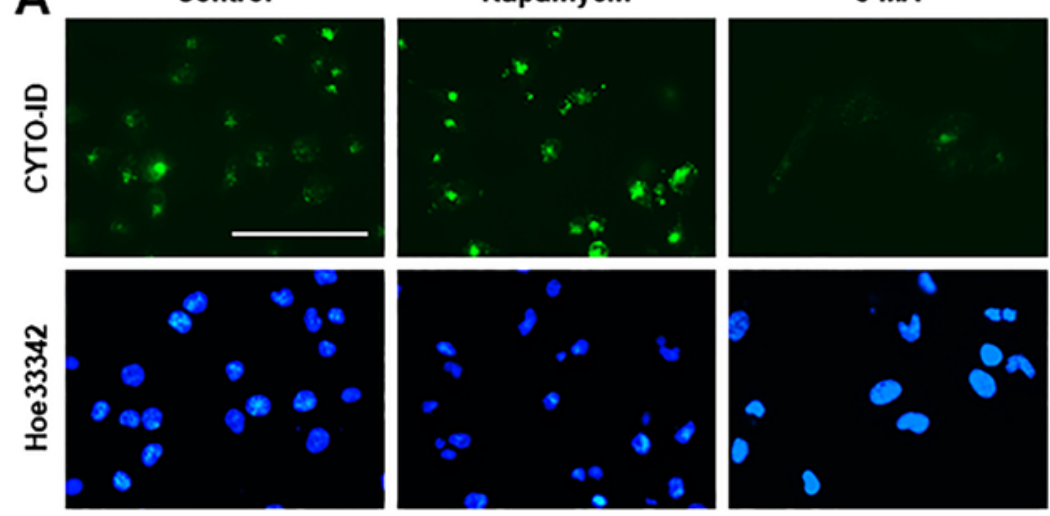

B

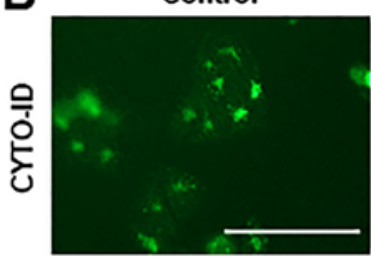

HOS
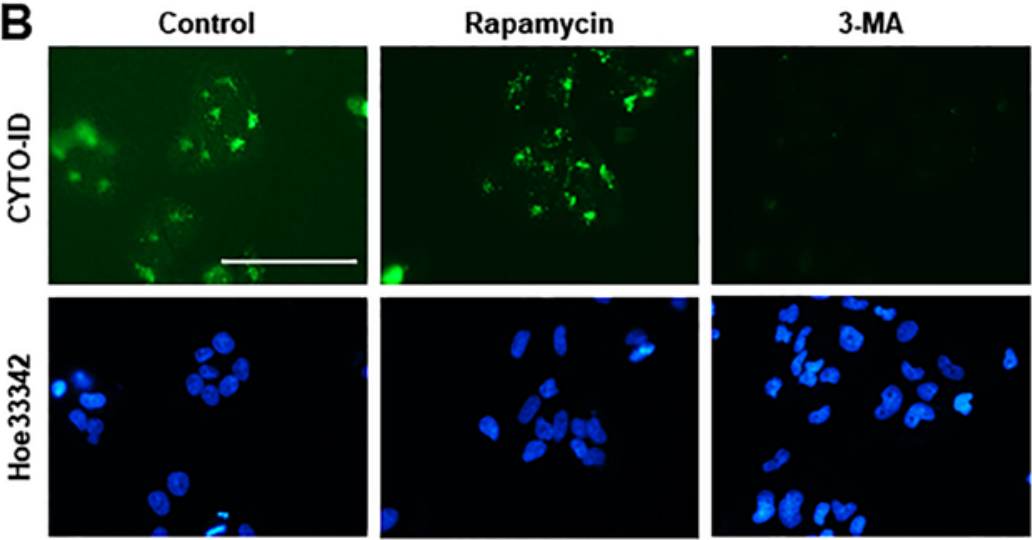

Figure 1. Human malignant melanoma and osteosarcoma cells exhibit substantial basal autophagy. (A) A375 and (B) HOS cells were treated with $0.5 \mu \mathrm{M}$ rapamycin or $5 \mathrm{mM} \mathrm{3-MA}$ for $24 \mathrm{~h}$ and were then stained with CYTO-ID for $1 \mathrm{~h}$ at $37^{\circ} \mathrm{C}$ in the dark. The nuclei were counterstained with Hoechst 33342 . Images were obtained using an EVOS FL Cell Imaging system. Scale bar, $100 \mu \mathrm{m}$. 3-MA, 3-methyladenine.

The results demonstrated that TRAIL increased CYTO-ID puncta in TRAIL-resistant A375 cells; however, TRAIL led to modest mitochondrial fragmentation, and caused minimal mitochondrial aggregation and colocalization with autophagosomes (Fig. 3C, lower panels). These results indicated that PSM may induce colocalization of aggregated mitochondria and autophagosomes.

Toxic PSM specifically increases autophagic flux. The present results indicated that PSM specifically leads to colocalization of aggregated mitochondria and autophagosomes. To determine whether this intrinsic event is cytoprotective or cytotoxic, the study examined the association of PSM with cell damage. PSM (100\%) displayed significant cytotoxic activity toward MM (A2058) and OS (HOS) cells, whereas it was ineffective at lower concentrations ( $\leq 50 \%$ ) (Fig. 4A and B). Subsequently, the present study compared the effects of toxic and subtoxic PSM on autophagic flux. The cytosolic protein LC3-I forms a complex with phosphatidylethanolamine, thus resulting in the formation of LC3-II, which directly associates with the autophagosome membrane. LC3-II enables the binding of adaptor proteins, such as p62/sequestome 1 (36). Therefore, the present study analyzed the expression levels of p62 and LC3-II following PSM treatment. Subtoxic PSM had minimal effects on the expression levels of p62 and LC3-II up to $72 \mathrm{~h}$ post-stimulation (Fig. 4C, left panels). However, toxic PSM increased the expression levels of LC3-II, but not p62, 36-48 h post-stimulation (Fig. 4C, right panels). These results suggested that toxic PSM may specifically increase autophagic flux.

Colocalization of mitochondria and autophagosomes is associated with autophagy induction and cell death. To further explore the relationship between the colocalization of mitochondria and autophagosomes and autophagy, the present study examined the effects of autophagy modulators on mitochondrial morphology and autophagosome location. Treatment with the autophagy promoter rapamycin markedly increased CYTO-ID puncta in HOS cells (Fig. 5A). Concomitantly, some, but not all, of the puncta were colocalized with the mitochondria, as shown by the appearance of yellow signals in the overlay images. Conversely, 3-MA completely suppressed CYTO-ID puncta and increased mitochondrial hyperfusion (Fig. 5A). In addition, subtoxic PSM did not induce robust colocalization of the mitochondria and autophagosomes (Fig. 5A). All of these agents caused minimal mitochondrial fragmentation and aggregation. Furthermore, toxic PSM strongly induced mitochondrial fragmentation and aggregation, and colocalization of mitochondria and autophagosomes (Fig. 5A). Microscopic analysis with a higher resolution confirmed these observations and indicated the colocalization of clustered CYTO-ID puncta and aggregated mitochondria (Fig. 5B). These results suggested that the colocalization of mitochondria and autophagosomes may be associated with autophagy induction and cell damage. 

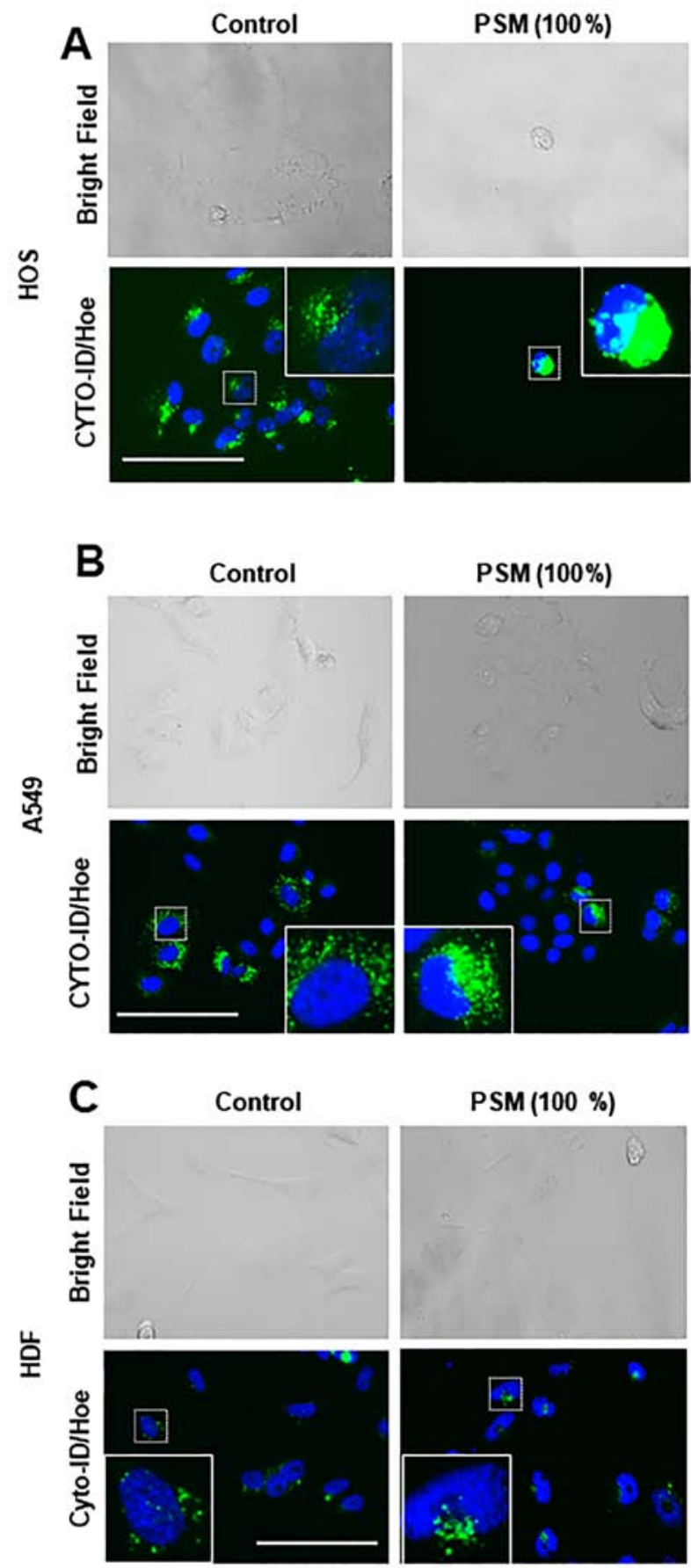

Figure 2. Clustering of CYTO-ID puncta accompanies cancer cell damage induced by PSM. (A) HOS, (B) A549 and (C) HDF cells were suspended in $10 \%$ FBS/DMEM, and once the cells became adherent, the medium was removed by aspiration. Subsequently, adherent cells were treated with an equal volume of PSM (100\%) and 10\% FBS/DMEM, and incubated for $24 \mathrm{~h}$ at $37^{\circ} \mathrm{C}$. Cells were stained with CYTO-ID and Hoechst 33342 . Images were obtained and analyzed using an EVOS FL Cell Imaging system. Scale bar, $100 \mu \mathrm{m}$. DMEM, Dulbecco's modified Eagle's medium; FBS, fetal bovine serum; PSM, cold plasma-stimulated medium.

PSM induces ACD during the initial 24 h post-stimulation. To further explore the role of autophagy in the antitumor activity of PSM, the present study examined the effects of numerous pharmacological autophagy inhibitors with various mechanisms of action on PSM cytotoxicity. 3-MA inhibits autophagosome formation by inhibiting the type III phosphatidylinositol-3-kinase, whereas the antimalarial drug
CQ inhibits the fusion of autophagosomes and lysosomes. The antibiotic Baf inhibits vacuolar-type $\mathrm{H}^{+}$-ATPases and increases lysosomal $\mathrm{pH}$, thus compromising the fusion of autophagosomes and lysosomes. HOS cells were relatively susceptible to PSM cytotoxicity. Accordingly, their viability often decreased considerably (maximum reduction, $80 \%$ ) at $24 \mathrm{~h}$ following treatment with PSM ( $\geq 25 \%$ ) (Fig. 6A). Baf inhibited the cytotoxic effects of $25 \%$ PSM entirely, and those of $100 \%$ PSM partially. In addition, 3-MA significantly reduced the effects of PSM (25\%), but not those of PSM (100\%). PSM (100\%), but not PSM (25\%), also significantly decreased the viability of SAOS-2 and MG63 cells (Fig. 6B and C), and 3-MA inhibited the cytotoxic effects of PSM in MG63 cells, but not in SAOS-2 cells, whereas Baf blocked the effects in both cell types. These results demonstrated that PSM may induce ACD during the initial $24 \mathrm{~h}$ post-stimulation.

PSM induces another reactive oxygen species (ROS)dependent cell death modality upon prolonged treatment. PSM exerted higher degrees of cytotoxicity toward MM and OS cells following prolonged treatment $(72 \mathrm{~h})$. As a result, PSM ( $\geq 25 \%$ ) markedy decreased the viability of HOS, 143B and A2058 cells (Fig. 7A-C). Notably, in all cell lines tested, these effects were not blocked by any of the autophagy inhibitors (3-MA, Baf and CQ) (Fig. 7A-C). In addition, 3-MA $(5 \mathrm{mM})$ and CQ $(100 \mu \mathrm{M})$ alone significantly reduced the viability of 143B cells. Previously, it was demonstrated that ROS serve a vital role in mediating PSM cytotoxicity $(30,37)$. As a result, the superoxide oxidase mimetic antioxidant MnTBaP has been revealed to suppress the effects of PSM in HOS cells, whereas the effects were more pronounced in cells treated with PSM (25\%) compared with in those treated with PSM (100\%) (37). In agreement with our previous observations (30), MnTBaP significantly reduced the cytotoxicity of PSM in the present study, with a higher potency toward the lower concentration of PSM (Fig. 7D). Conversely, the pancaspase-inhibitor z-VAD-FMK exhibited minimal effects on PSM cytotoxicity regardless of the PSM concentration and cell type examined (data not shown). These results indicated that PSM may also induce another ROS-dependent, non-apoptotic, non-autophagic cell death upon prolonged treatment.

TRAIL predominantly triggers cytoprotective autophagy, which prevents apoptotic and non-apoptotic cell death. Since TRAIL increased CYTO-ID puncta in cancer cells, the present study explored the possible role of autophagic flux in the antitumor activity of TRAIL. A 72-h-incubation protocol was employed, because cells were highly TRAIL-resistant and TRAIL treatment for $24 \mathrm{~h}$ had minimal cytotoxicity. The autophagy inhibitors 3 -MA $(\geq 1.3 \mathrm{mM})$ and CQ $(\geq 100 \mu \mathrm{M})$ alone dose-dependently decreased the viability of A2058 and SAOS-2 cells. Furthermore, the low concentration of 3-MA $(1.3 \mathrm{mM})$ significantly potentiated the cytotoxicity of TRAIL in the two cell lines (Fig. 8A and B). Conversely, $\leq 300 \mathrm{nM}$ Baf neither decreased cell viability nor enhanced the effect of TRAIL, regardless of the cell type examined. The concentration of 3-MA suitable for TRAIL sensitization varied depending on the cell lines tested (i.e., HOS and SAOS-2 cells), z-VAD-FMK entirely suppressed the effect. Z-VAD-FMK significantly inhibited the effects of 3-MA (2.5 mM) on HOS 


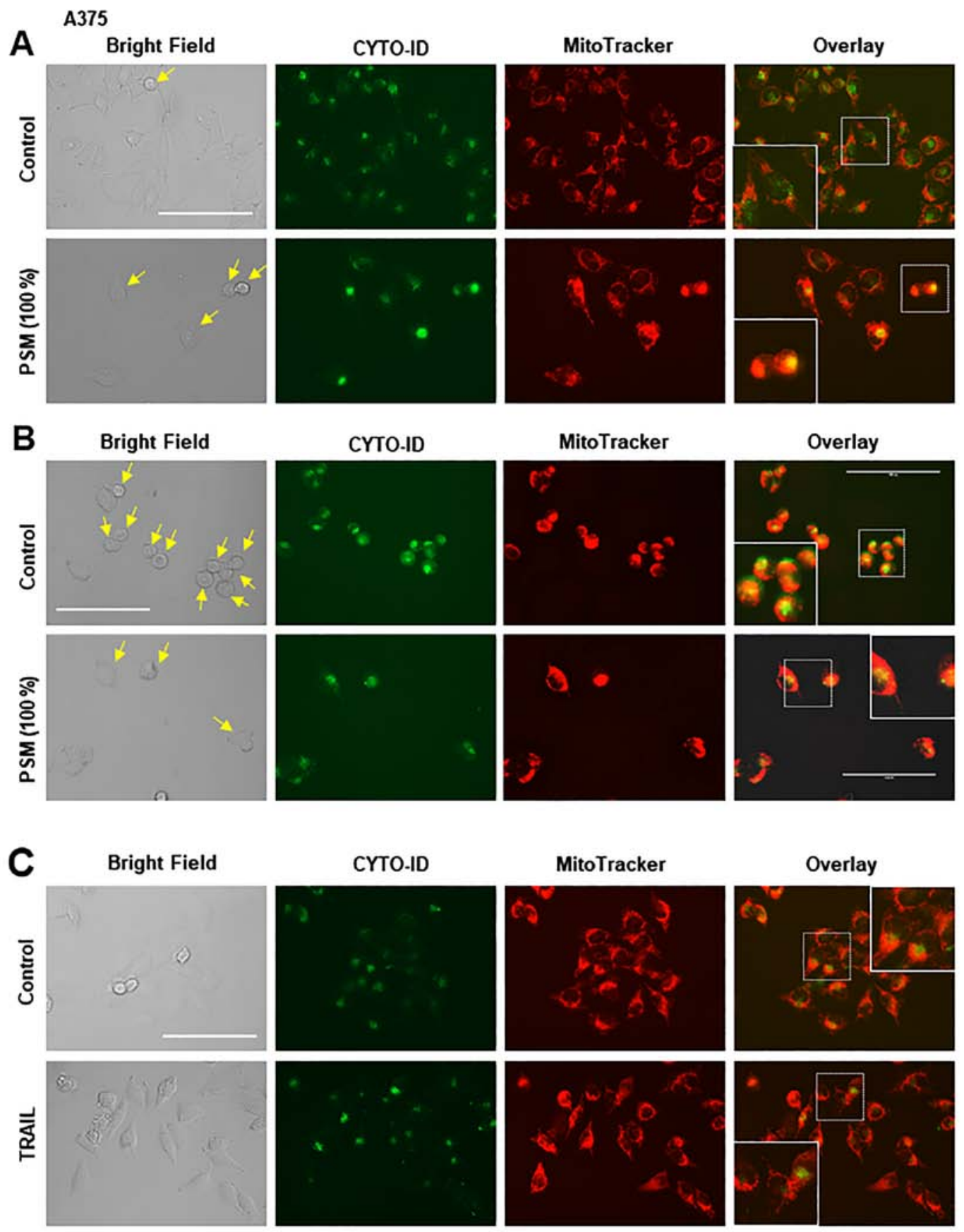

Figure 3. PSM specifically induces colocalization of aggregated mitochondria and CYTO-ID puncta. (A and C) Normally grown and (B) overgrown A375 cells were treated with (A and B) PSM (100\%) or (C) $100 \mathrm{ng} / \mathrm{ml}$ TRAIL for $24 \mathrm{~h}$ at $37^{\circ} \mathrm{C}$, and were stained with CYTO-ID and MitoTracker Red CMXRos. In the panels labeled 'Overlay', the images in the white boxes with solid lines represent the expansion of images in the smaller boxes with dotted lines. Images were obtained and analyzed using an EVOS FL Cell Imaging system. Scale bar, $100 \mu \mathrm{m}$. PSM, cold plasma-stimulated medium.

cells. In addition, z-VAD-FMK completely blocked the effects of 3-MA (1.3 mM) and minimally inhibited the effects of 3-MA (2.5 mM) on SAOS-2 cells (Fig. 8C and D). Furthermore, the present study analyzed the effects of cotreatment with TRAIL and 3-MA on autophagy and mitochondrial morphology. TRAIL alone increased CYTO-ID puncta, whereas 3-MA alone abolished them in HOS cells (Fig. 8E). Concomitantly, mitochondria became hyperfused in response to TRAIL or 3-MA, as shown by increased highly intra-connected mitochondria (Fig. 8E). The combined application of TRAIL and 3-MA led to the formation of large clusters of CYTO-ID puncta that colocalized with the mitochondria (Fig. 8E). Collectively, these results indicated that TRAIL predominantly triggers cytoprotective autophagy, the prevention of which may lead to colocalization of the mitochondria and autophagosomes, apoptosis and another form of non-apoptotic cell death.

\section{Discussion}

The present study aimed to determine whether PSM modulated autophagy in human MM and OS cells. Western blotting demonstrated that toxic, but not subtoxic, PSM increased LC3-II expression, which is required for autophagosome formation. Furthermore, the cytotoxic effects of PSM were 
A

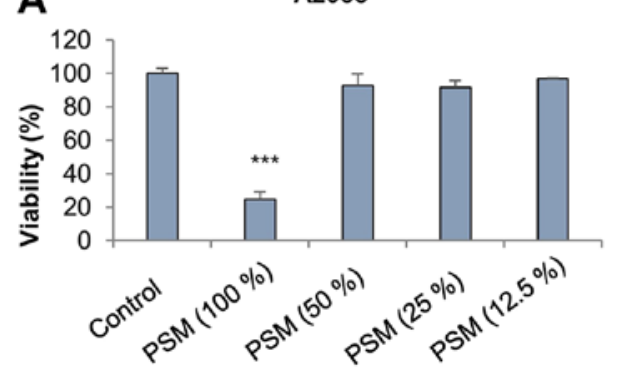

C $143 \mathrm{~B}$ 43B p62

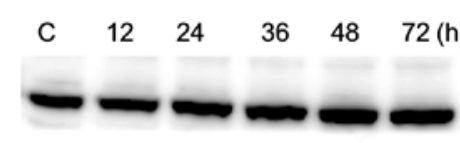

LC3-1/II

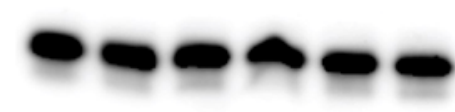

GAPDH

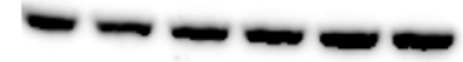

B

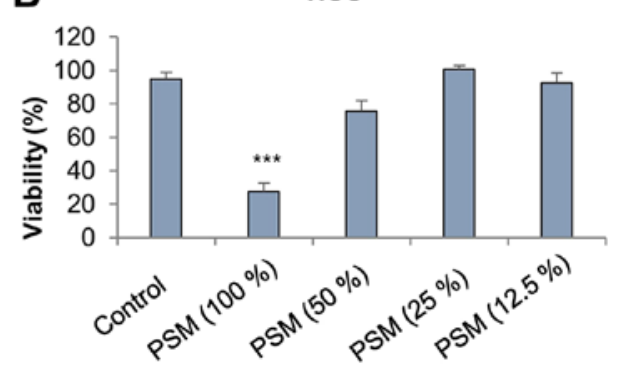

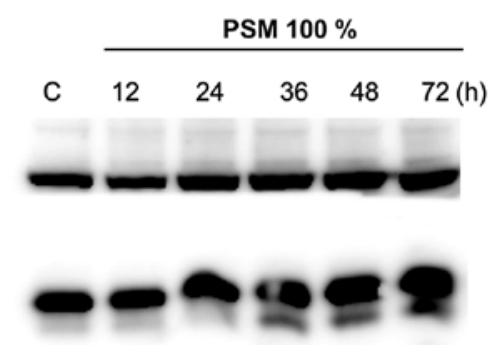

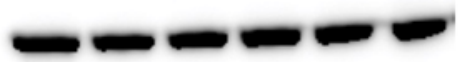

Figure 4. Toxic PSM increases autophagic flux. (A) A2058 and (B) HOS cells were treated with various concentrations of PSM for 72 h. Subsequently, cell viability was analyzed using the WST- 8 assay in triplicate. Data are presented as the means \pm standard deviation of a representative experiment ( $=3$ ). (C) 143B cells were treated with PSM (25 and 100\%) for 12, 24, 36, 48 and $72 \mathrm{~h}$ at $37^{\circ} \mathrm{C}$. Western blotting was used to detect p62, LC3 and GAPDH expression. LC3, microtubule-associated protein 1A/1B-light chain 3; PSM, cold plasma-stimulated medium.

strongly inhibited following treatment with the pharmacological autophagy inhibitors 3-MA and Baf, thus indicating that autophagy is responsible for cell death. These findings suggested that PSM may trigger ACD in tumor cells. Notably, mitochondrial abnormalities accompanied the induction of ACD. In agreement with our previous study (30), PSM induced mitochondrial aberrations in a concentration-dependent manner. Toxic PSM led to excessive mitochondrial fragmentation and aggregation, whereas subtoxic PSM induced only modest mitochondrial fragmentation. In addition, live-cell imaging of CYTO-ID puncta revealed marked alterations in the status and location of autophagosomes in response to PSM. When unstimulated, autophagosomes existed as small diffuse particles, the majority of which were localized in the cytoplasm separate from the mitochondria. However, following PSM treatment, autophagosomes formed a cluster that colocalized with the aggregated mitochondria. In mitophagy, damaged mitochondria are engulfed by autophagosomes, so that mitochondria and autophagosomes eventually colocalize. Therefore, the specific effects of toxic PSM appear similar to mitophagy. Our previous study demonstrated that mitochondria were heavily damaged, resulting in the loss of mitochondrial membrane potential $\left(\Delta \Psi_{\mathrm{m}}\right)$ and integrity, when they became punctate and aggregated $(32,33)$. Notably, dissipation of the $\Delta \Psi_{\mathrm{m}}$ is a primary trigger of mitophagy (34). Diverse insults, such as carbonyl cyanide m-chlorophenylhydrazone, carbonyl cyanide p-triflouromethoxyphenylhydrazone and salinomycin, commonly induce loss of the $\Delta \Psi_{\mathrm{m}}$, thereby activating mitophagy $(38,39)$. Loss of the $\Delta \Psi_{\mathrm{m}}$ leads to the accumulation of phosphatase and tensin homolog-induced putative kinase 1 (PINK1) on the outer mitochondrial membrane. The localized PINK1 phosphorylates E3-ubiquitin ligase Parkin and activates Parkin-mediated ubiquitination, thus resulting in autophagic degradation of the damaged organelles (34). Therefore, it is possible that PSM injures the mitochondria, thereby leading to mitophagy in the present cell system. p62 has been reported to serve a vital role in mitophagy (40), whereas it is superfluous in some circumstances (41). The present study demonstrated that only marginal p62 accumulation occurred following PSM treatment; therefore, the role of p62 in autophagic flux appears to be minor in the present cell system. Several lines of evidence indicated that the mitophagylike event observed promoted cell death and contributed to the antitumor activity of PSM: i) The event spontaneously occurred in damaged or dying cancer cells; ii) TRAIL, which had only a modest cytotoxic effect, did not induce the event; iii) toxic, but not subtoxic PSM, caused the event; (iv) TRAIL and 3-MA synergistically increased the event and cell killing. It is widely accepted that controlled mitophagy is cytoprotective, since it removes damaged mitochondria; however, excessive mitophagy leads to cell damage by compromising the energy supply, and $\mathrm{Ca}^{2+}$ and metabolic homeostasis. Therefore, it is possible that PSM may induce excessive mitophagy, which is responsible for the antitumor activity of PSM. However, further studies are required to verify this hypothesis.

The present data indicated that a basal level of autophagy operates in MM and OS cells; these cells possessed substantial levels of autophagosomes even under nutritional and stressfree conditions. Compared with the mitophagy-like event, basal autophagy appeared to serve a cytoprotective role, since 


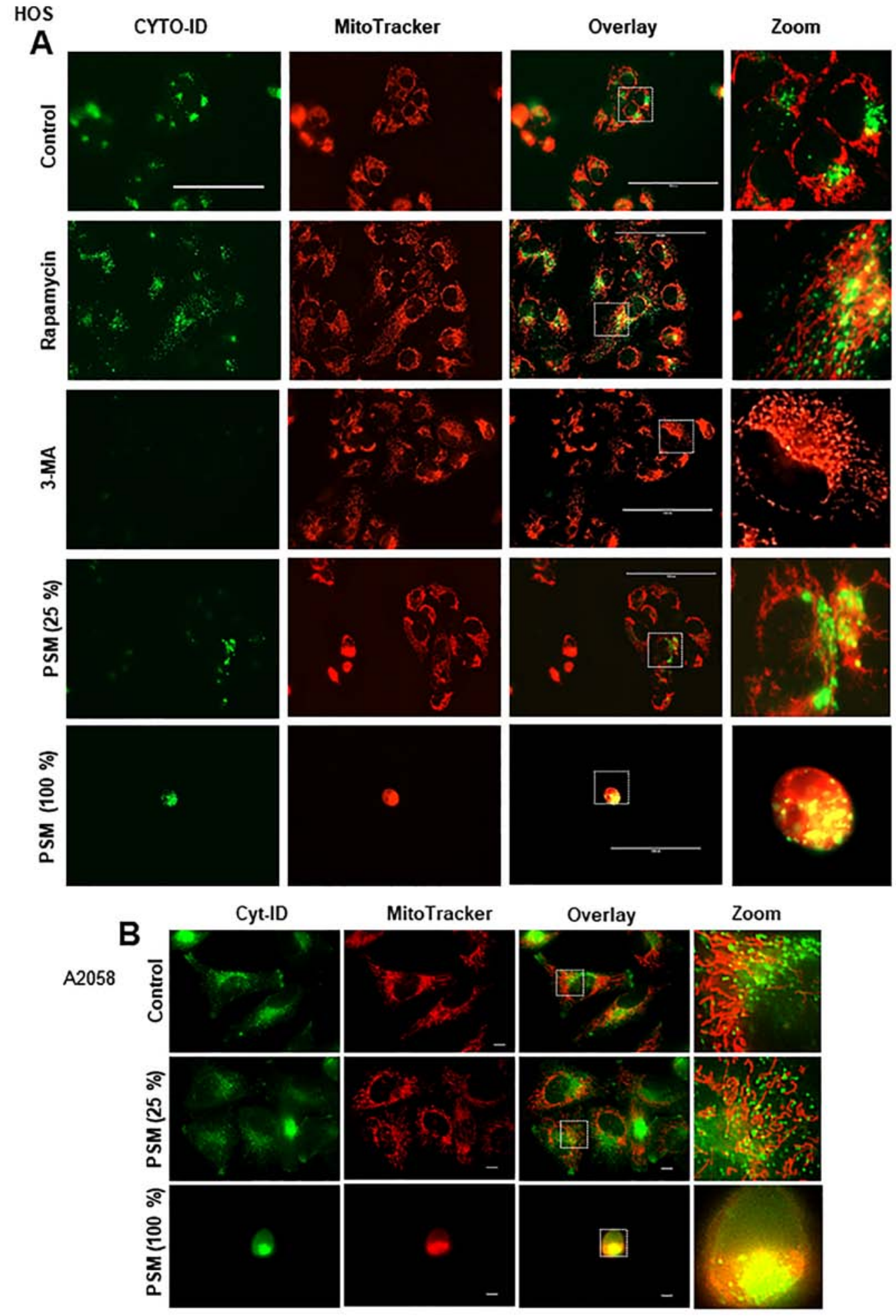

Figure 5. Colocalization of the mitochondria and autophagosomes is associated with the induction of autophagy and cell damage. (A) HOS cells were treated with $1 \mu \mathrm{M}$ rapamycin, $5 \mathrm{mM}$ 3-MA and PSM (25 and 100\%) for $24 \mathrm{~h}$, and were stained with CYTO-ID and MitoTracker Red CMXRos. Panels labeled 'Zoom' represent expansion of the small white boxes within the 'Overlay' images. Scale bar, $100 \mu \mathrm{m}$. (B) A2058 cells were treated with PSM (25 or $100 \%)$ for $24 \mathrm{~h}$, and were stained with CYTO-ID and MitoTracker Red CMXRos. Images were obtained using a BZ X-700 Fluorescence Microscope equipped with a 100X coverslip-corrected oil objective. Images were analyzed using BZ-H3A application software and ImageJ software. The panels labeled 'Zoom' represent the expansion of the small white boxes within 'Overlay' images. Scale bar, $10 \mu \mathrm{m}$. 3-MA, 3-methyladenine; PSM, cold plasma-stimulated medium.

autophagy inhibitors, including 3-MA and CQ alone exerted significant cytotoxicity toward these cancer cells in the absence of insult. Notably, the effects were more pronounced following $72 \mathrm{~h}$ treatment compared with after $24 \mathrm{~h}$. These observations indicated that basal autophagy may be essential for cell survival, particularly in response to hypo-nutritional conditions. The present study suggested that in these cancer cells, TRAIL primarily induced cytoprotective autophagy, since TRAIL increased autophagosome formation, whereas its cytotoxicity was amplified, rather than inhibited, following treatment with 
A

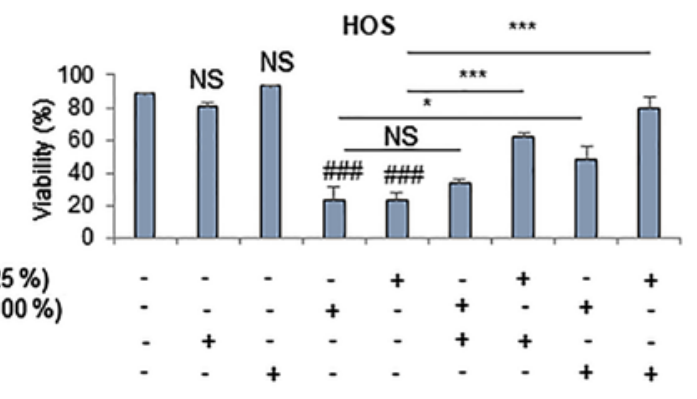

B

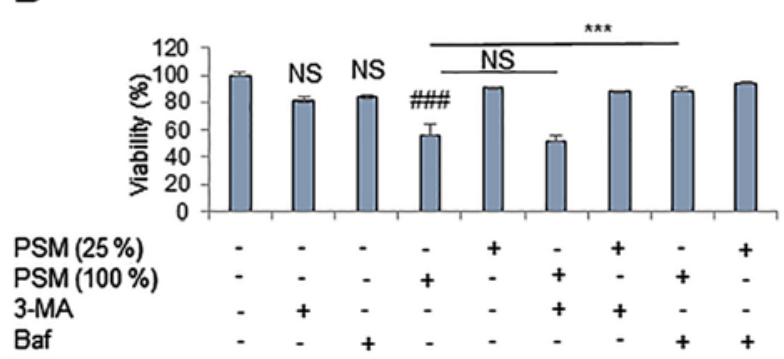

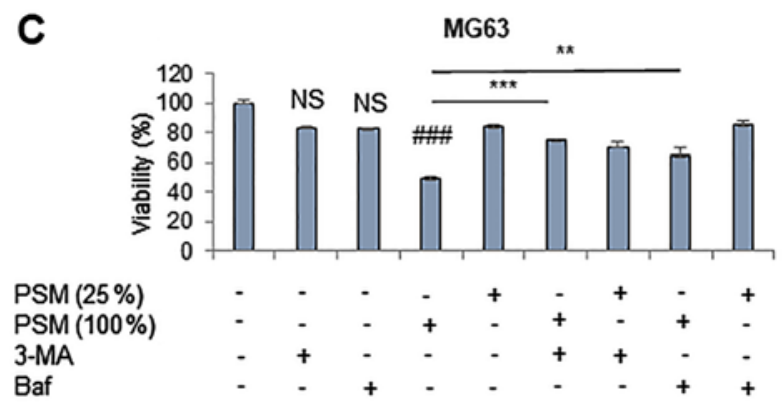

Figure 6. PSM induces ACD during the initial 24 h post-stimulation. (A) HOS, (B) SAOS-2 and (C) MG63 cells were treated with PSM (25 and $100 \%$ ), $5 \mathrm{mM}$ 3-MA and $100 \mathrm{nM}$ Baf alone or in combination for $24 \mathrm{~h}$, and cell viability was analyzed using WST-8 assay in triplicate. Data are presented as the means \pm standard deviation of a representative experiment $(n=3)$. Data were analyzed by one-way analysis of variance followed by Tukey's post hoc test.

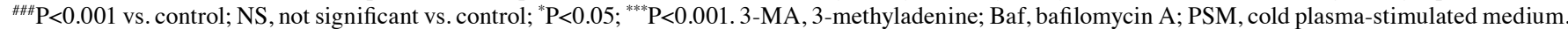
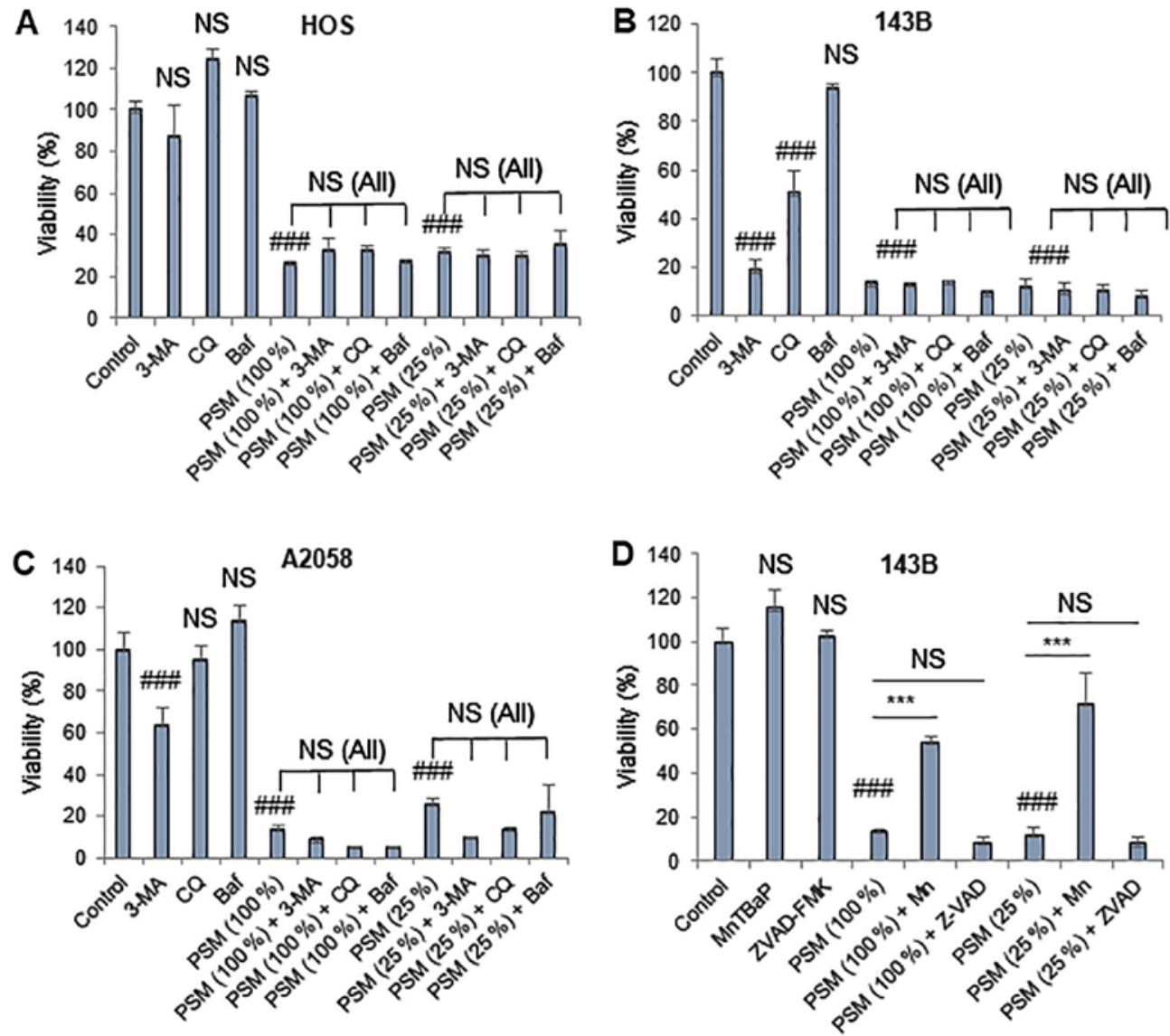

Figure 7. PSM induces another ROS-dependent cell death modality upon prolonged treatment. (A) HOS, (B and D) 143B and (C) A2058 cells were treated with (A-C) PSM (25 and 100\%) and $5 \mathrm{mM} 3-\mathrm{MA}, 100 \mu \mathrm{M} \mathrm{CQ}$ and $100 \mathrm{nM}$ Baf alone or in combination for $72 \mathrm{~h}$. (D) $143 \mathrm{~B}$ cells were treated with PSM (25 and $100 \%)$ and $10 \mu \mathrm{M} \mathrm{ZVAD}$ and $30 \mu \mathrm{M}$ Mn alone or in combination for $72 \mathrm{~h}$. Cell viability was analyzed using the WST-8 assay in triplicate. Data are presented as the means \pm standard deviation of a representative experiment $(n=3)$. Data were analyzed by analysis of variance followed by Tukey's post hoc test. ${ }^{\# \#} \mathrm{P}<0.001$ vs. control; NS, not significant vs. control; ${ }^{* * * *} \mathrm{P}<0.001$. NS (All), no statistical significance between PSM in combination with 3-MA, CQ and Baf compared with PSM (25 and 100\%) alone. 3-MA, 3-methyladenine; Baf, bafilomycin A; CQ, chloroquine; Mn, MnTBaP; PSM, cold plasma-stimulated medium; ZVAD, z-VAD-fluorometheylketone. 
A

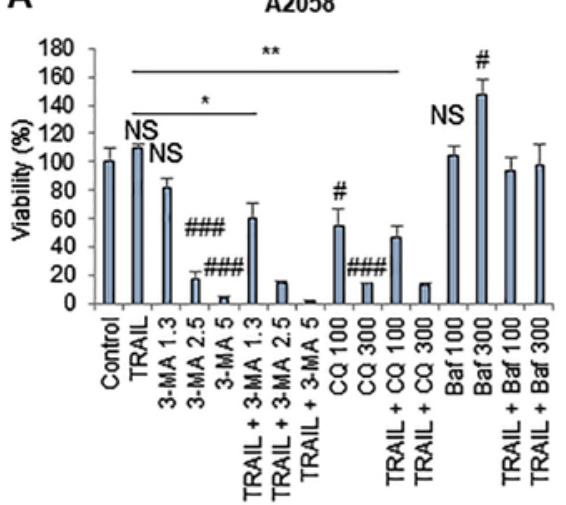

C

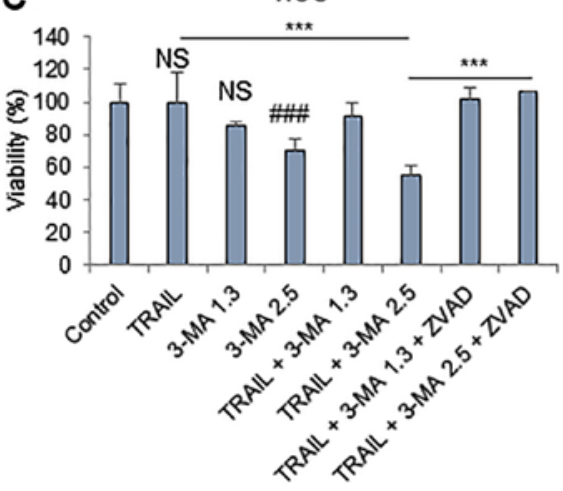

B
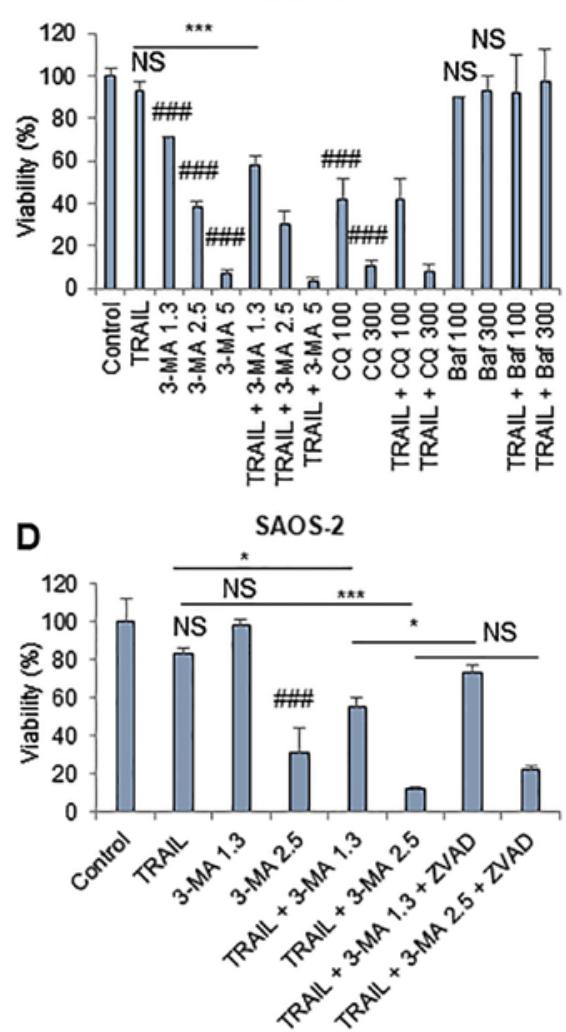

E HOS

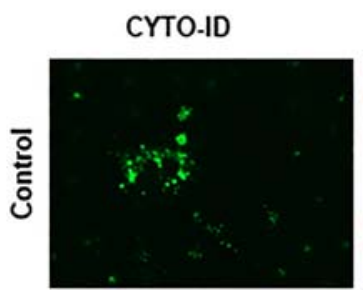

MitoTracker
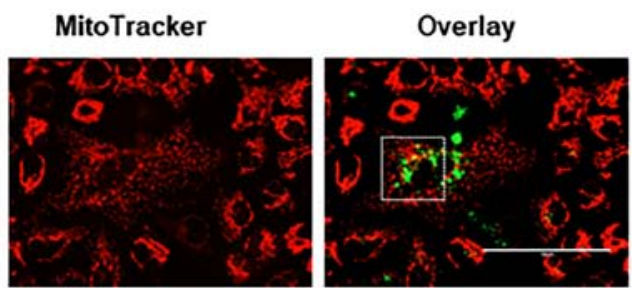

Zoom
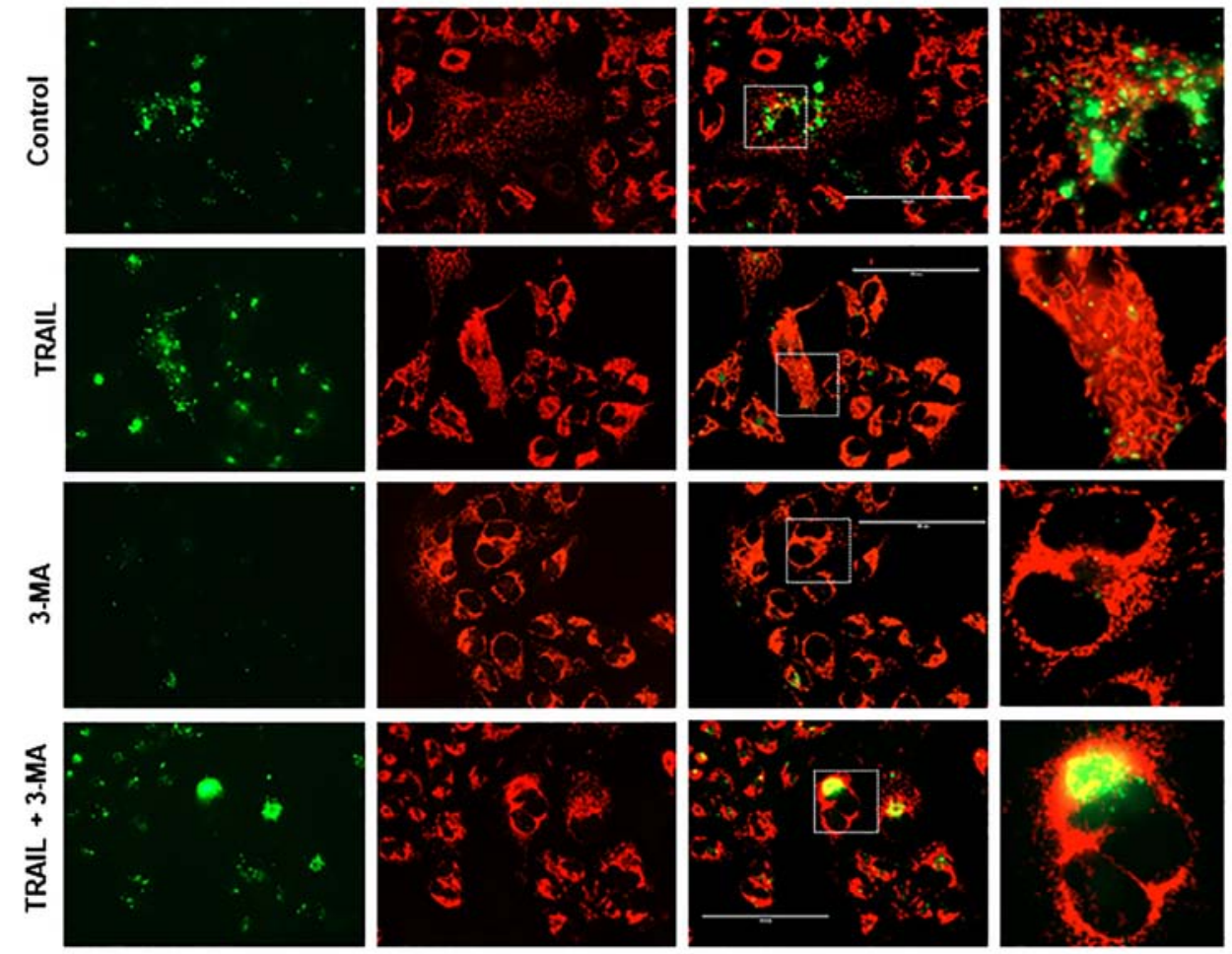

Figure 8. TRAIL predominantly triggers cytoprotective autophagy. (A) A2058 and (B) SAOS-2 cells were treated with $100 \mathrm{ng} / \mathrm{ml}$ TRAIL, 3-MA, CQ and Baf at the indicated concentrations (3-MA, mM; CQ, $\mu \mathrm{M} ; \mathrm{Baf}, \mathrm{nM}$ ) alone or in combination for $72 \mathrm{~h}$. (C) HOS and (D) SAOS-2 cells were treated with $100 \mathrm{ng} / \mathrm{ml}$ TRAIL and 3-MA at the indicated concentrations (mM) for $72 \mathrm{~h}$, in the absence or presence of $10 \mu \mathrm{M}$ ZVAD. Cell viability was analyzed using the WST-8 assay in triplicate. Data are presented as the means \pm standard deviation of a representative experiment $(n=3)$. Data were analyzed by analysis of variance followed by Tukey's post hoc test. ${ }^{\# \# \#} \mathrm{P}<0.001 ;{ }^{\#} \mathrm{P}<0.05$ vs. control; NS, not significant. ${ }^{* * *} \mathrm{P}<0.001 ;{ }^{* *} \mathrm{P}<0.01 ;{ }^{*} \mathrm{P}<0.05$. (E) HOS cells were treated with $100 \mathrm{ng} / \mathrm{ml}$ TRAIL and $5 \mathrm{mM}$ 3-MA alone or in combination, and were stained with CYTO-ID and MitoTracker Red CMXRos. Panels labeled 'Zoom' represent the expansion of the small while boxes within 'Overlay' images. Scale bar, $100 \mu \mathrm{m}$. 3-MA, 3-methyladenine; Baf, bafilomycin A; CQ, chloroquine; Mn, MnTBaP; PSM, cold plasma-stimulated medium; ZVAD, z-VAD-fluorometheylketone. 
autophagy inhibitors, including 3-MA. Notably, 3-MA/CQ and Baf had various effects on cell viability and TRAIL cytotoxicity. At present, the reason for the results are unclear, because they commonly serve as autophagy inhibitors. However, it was previously demonstrated that 3-MA, but not Baf, interferes with mitochondrial $\mathrm{Ca}^{2+}$ loading, which is an essential process for energy production and cell survival. These observations are intriguing, since our recent study demonstrated that disturbance of mitochondrial $\mathrm{Ca}^{2+}$ may lead to cancer cell death and sensitization to TRAIL cytotoxicity (31). Therefore, it is possible that the difference resides in autophagy-independent effects. Further investigations are required.

The results of the present study are consistent with those previously reported in various cancer cell types, including lung, bladder, prostate, colorectal cancer and hepatoma cells (42-44). Notably, despite its ability to trigger such cytoprotective autophagy, TRAIL minimally led to the putative cytotoxic mitophagy-like event. Therefore, TRAIL may be similar to various antitumor drugs, such as temozolomide, epirubicin and sorafenib, which have been reported to engage cytoprotective autophagy (45-48). Furthermore, the blockade of basal autophagy by 3-MA enabled TRAIL to increase the mitophagy-like event and kill TRAIL-resistant MM and OS cells, whereas it promoted cell death, but not ACD. In addition, the mitochondrial morphological alterations observed in response to TRAIL + 3-MA appeared to be somewhat different from those observed following toxic PSM treatment. Collectively, these results suggested that suppression of basal cytoprotective autophagy may promote the cytocidal mitophagy-like event, whereas another fundamental cellular event is required for induction of the full mitochondrial abnormalities leading to ACD induction. Further studies are required to identify such events.

Recent evidence has indicated that autophagy and apoptosis are closely associated, and that they may regulate one another (49-51). This connection has a significant impact on tumor cell sensitivity to antitumor chemotherapeutic drugs. In particular, negative regulation of apoptosis by autophagy is significant, since various antitumor drugs have been revealed to induce cytoprotective autophagy, which allows cancer cells to cope with apoptotic insults. Accordingly, the induction of autophagy in cancer cells represents a double-edged sword, leading to cell survival or ACD depending on the cell type and conditions. Therefore, it is critical to understand the pathways controlling context-dependent autophagy for the application of ACD in cancer treatment.

In conclusion, the present study demonstrated that PSM may trigger cytocidal autophagy, whereas TRAIL triggered cytoprotective autophagy. To the best of our knowledge, the present study is the first to demonstrate that PSM may induce ACD in human cancer cells. These findings provide a rationale for the development of PSM as a novel approach for the treatment of apoptosis-resistant tumors, including MM and OS. In addition, PSM may serve as a useful model for studying the mechanisms underlying ACD induction in cancer cells.

\section{Acknowledgements}

The authors would like to thank the JCRB Cell Bank of National Institutes of Biomedical Innovation, Health and
Nutrition (Osaka, Japan) and Riken BioResource Center (Tsukuba, Japan) for providing cell lines.

\section{Funding}

This work was supported in part by JSPS KAKENHI Grant Number 15K09750 to YSK and 15K09792 to TO.

\section{Availability of data and materials}

The datasets used and/or analyzed during the current study are available from the corresponding author on reasonable request

\section{Authors' contributions}

TI performed experiments, analyzed data and wrote the manuscript. TA performed experiments and wrote the manuscript. MSK and TT performed experiments and analyzed data. YY, TO and YT conceived and designed the study, performed the critical revision of the manuscript, and provided analytical tools. YSK conceived and designed the study, performed experiments and wrote the manuscript. All authors read and approved the final manuscript.

\section{Ethics approval and consent to participate}

Not applicable.

\section{Consent for publication}

Not applicable.

\section{Conflict of interest}

The authors declare that they have no competing interests.

\section{References}

1. Almasan A and Ashkenazi A: Apo2L/TRAIL: Apoptosis signaling, biology, and potential for cancer therapy. Cytokine Growth Factor Rev 14: 337-348, 2003

2. Johnstone RW, Frew AJ and Smyth MJ: The TRAIL apoptotic pathway in cancer onset, progression and therapy. Nat Rev Cancer 8: 782-798, 2008.

3. Wang S: The promise of cancer therapeutics targeting the TNF-related apoptosis-inducing ligand and TRAIL receptor pathway. Oncogene 27: 6207-6215, 2008.

4. Gonzalvez F and Ashkenazi A: New insights into apoptosis signaling by Apo2L/TRAIL. Oncogene 29: 4752-4765, 2010.

5. Kischkel FC, Lawrence DA, Chuntharapai A, Schow P, Kim KJ and Ashkenazi A: Apo2L/TRAIL-dependent recruitment of endogenous FADD and caspase- 8 to death receptors 4 and 5. Immunity 12: 611-620, 2000.

6. LeBlanc HN and Ashkenazi A: Apo2L/TRAIL and its death and decoy receptors. Cell Death Differ 10: 66-75, 2003.

7. Ivanov VN, Bhoumik A and Ronai Z: Death receptors and melanoma resistance to apoptosis. Oncogene 22: 3152-3161, 2003.

8. Dyer MJ, MacFarlane M and Cohen GM: Barriers to effective TRAIL-targeted therapy of malignancy. J Clin Oncol 25: 25: 4505-4506, 2007.

9. Dimberg LY, Anderson CK, Camidge R, Behbakht K, Thorburn A and Ford HL: On the TRAIL to successful cancer therapy? Predicting and counteracting resistance against TRAIL-based therapeutics. Oncogene 32: 1341-1350, 2013.

10. Guiho R, Biteau K, Heymann D and Redini F: TRAIL-based therapy in pediatric bone tumors: How to overcome resistance. Future Oncol 11: 535-542, 2015. 
11. de Miguel D, Lemke J, Anel A, Walczak H and MartinezLostao L: Onto better TRAILs for cancer treatment. Cell Death Differ 23: 733-747, 2016.

12. Keidar M, Walk R, Shashurin A, Srinivasan P, Sandler A Dasgupta S, Ravi R, Guerrero-Preston R and Trink B: Cold plasma selectivity and the possibility of a paradigm shift in cancer therapy. Br J Cancer 105: 1295-1301, 2011.

13. Zucker SN, Zirnheld J, Bagati A, DiSanto TM, Des Soye B, Wawrzyniak JA, Etemadi K, Nikiforov M and Berezney R: Preferential induction of apoptotic cell death in melanoma cells as compared with normal keratinocytes using a non-thermal plasma torch. Cancer Biol Ther 13: 1299-1306, 2012.

14. Arndt S, Unger P, Wacker E, Shimizu T, Heinlin J, Li YF, Thomas HM, Morfill GE, Zimmermann JL, Bosserhoff AK, et al: Cold atmospheric plasma (CAP) changes gene expression of key molecules of the wound healing machinery and improves wound healing in vitro and in vivo. PLoS One 8: e79325, 2013.

15. Ishaq M, Evans MM and Ostrikov KK: Effect of atmospheric gas plasmas on cancer cell signaling. Int J Cancer 134: 1517-1528, 2014.

16. Utsumi F, Kajiyama H, Nakamura K, Tanaka H, Mizuno M, Ishikawa K, Kondo H, Kano H, Hori M and Kikkawa F: Effect of indirect nonequilibrium atmospheric pressure plasma on antiproliferative activity against chronic chemo-resistant ovarian cancer cells in vitro and in vivo. PLoS One 8: e81576, 2013.

17. Torii K, Yamada S, Nakamura K, Tanaka H, Kajiyama H, Tanahashi K, Iwata N, Kanda M, Kobayashi D, Tanaka C, et al: Effectiveness of plasma treatment on gastric cancer cells. Gastric Cancer 18: 635-643, 2015.

18. Hattori N, Yamada S, Torii K, Takeda S, Nakamura K, Tanaka H, Kajiyama H, Kanda M, Fujii T, Nakayama G, et al: Effectiveness of plasma treatment on pancreatic cancer cells. Int J Oncol 47: $1655-1662,2015$

19. Adachi T, Tanaka H, Nonomura S, Hara H, Kondo S and Hori M: Plasma-activated medium induces A549 cell injury via a spiral apoptotic cascade involving the mitochondrial-nuclear network. Free Radic Biol Med 79: 28-44, 2015.

20. Ouyang L, Shi Z, Zhao S, Wang FT, Zhou TT, Liu B and Bao JK: Programmed cell death pathways in cancer: A review of apoptosis, autophagy and programmed necrosis. Cell Prolif 45: 487-498, 2012.

21. Maiuri MC, Zalckvar E, Kimchi A and Kroemer G: Self-eating and self-killing: Crosstalk between autophagy and apoptosis. Nat Rev Mol Cell Biol 8: 741-752, 2007.

22. Mizushima N, Yoshimori T and Ohsumi Y: The role of Atg proteins in autophagosome formation. Annu Rev Cell Dev Biol 27: 107-132, 2011.

23. Codogno P and Meijer AJ: Autophagy and signaling: Their role in cell survival and cell death. Cell Death Differ 12 (Suppl 2): $1509-1518,2005$

24. Díaz-Troya S, Pérez-Pérez ME, Florencio FJ and Crespo JL: The role of TOR in autophagy regulation from yeast to plants and mammals. Autophagy 4: 851-865, 2008

25. Dennis MD, Baum JI, Kimball SR and Jefferson LS: Mechanisms involved in the coordinate regulation of $\mathrm{mTORC} 1$ by insulin and amino acids. J Biol Chem 286: 8287-8296, 2011.

26. Bhutia SK, Mukhopadhyay S, Sinha N, Das DN, Panda PK, Patra SK, Maiti TK, Mandal M,Dent P, Wang XY, et al: Autophagy: Cancer's friend or foe? Adv Cancer Res 118: 61-95, 2013.

27. Gozuacik D and Kimchi A: Autophagy as a cell death and tumor suppressor mechanism. Oncogene 23: 2891-2906, 2004.

28. Fulda $S$ and Kögel D: Cell death by autophagy: Emerging molecular mechanisms and implications for cancer therapy. Oncogene 34: 5105-5113, 2015.

29. Fulda S: Autophagy in cancer therapy. Front Oncol 7: 128, 2017.

30. Saito K, Asai T, Fujiwara K, Sahara J, Koguchi H, Fukuda N, Suzuki-Karasaki $M$, Soma $M$ and Suzuki-Karasaki Y: Tumor-selective mitochondrial network collapse induced by atmospheric gas plasma-activated medium. Oncotarget 7: 19910$19927,2016$.

31. Takata N, Ohshima Y, Suzuki-Karasaki M, Yoshida Y, Tokuhashi Y and Suzuki-Karasaki Y: Mitochondrial $\mathrm{Ca}^{2+}$ removal amplifies TRAIL cytotoxicity toward apoptosis-resistant tumor cells via promotion of multiple cell death modalities. Int J Oncol 51: 193-203, 2017.

32. Suzuki-Karasaki Y, Fujiwara K, Saito K, Suzuki-Karasaki M, Ochiai T and Soma M: Distinct effects of TRAIL on the mitochondrial network in human cancer cells and normal cells: Role of plasma membrane depolarization. Oncotarget 6: 21572-21588, 2015.
33. Akita M, Suzuki-Karasaki M, Fujiwara K, Nakagawa C, Soma M, Yoshida Y, Ochiai T, Tokuhashi Y and Suzuki-Karasaki Y: Mitochondrial division inhibitor-1 induces mitochondrial hyperfusion and sensitizes human cancer cells to TRAIL-induced apoptosis. Int J Oncol 45: 1901-1912, 2014.

34. Kulikov AV, Luchkina EA, Gogvadze V and Zhivotovsky B: Mitophagy: Link to cancer development and therapy. Biochem Biophys Res Commun 482: 432-439, 2017.

35. Bordi M, Nazio F and Campello S: The close interconnection between mitochondrial dynamics and mitophagy in cancer. Front Oncol 7: 81, 2017.

36. Lystad AH, Ichimura Y, Takagi K, Yang Y, Pankiv S, Kanegae Y, Kageyama S, Suzuki M, Saito I, Mizushima T, et al: Structural determinants in GABARAP required for the selective binding and recruitment of ALFY to LC3B-positive structures. EMBO Rep 15: 557-565, 2014.

37. Tokunaga T,Ando T,Suzuki-Karasaki M,Ito T, Onoe-Takahashi A, Ochiai T, Soma M and Suzuki-Karasaki Y: Plasma-stimulated medium kills TRAIL-resistant human malignant cells by promoting caspase-independent cell death via membrane potential and calcium dynamics modulation. Int J Oncol 52: 697-708, 2018.

38. Narendra DP, Jin SM, Tanaka A, Suen DF, Gautier CA, Shen J, Cookson MR and Youle RJ: PINK1 is selectively stabilized on impaired mitochondria to activate Parkin. PLoS Biol 8: e1000298, 2010.

39. Jangamreddy JR, Ghavami S, Grabarek J, Kratz G, Wiechec E, Fredriksson BA, Rao Pariti RK, Cieślar-Pobuda A, Panigrahi S and $Ł o s$ MJ: Salinomycin induces activation of autophagy, mitophagy and affects mitochondrial polarity: Differences between primary and cancer cells. Biochim Biophys Acta 1833: 2057-2069, 2013.

40. Geisler S, Holmström KM, Skujat D, Fiesel FC, Rothfuss OC, Kahle PJ and Springer W: PINK1/Parkin-mediated mitophagy is dependent on VDAC1 and p62/SQSTM1. Nat Cell Biol 12: 119-131, 2010.

41. Narendra D, Kane LA, Hauser DN, Fearnley IM and Youle RJ: p62/SQSTM1 is required for Parkin-induced mitochondrial clustering but not mitophagy; VDAC1 is dispensable for both. Autophagy 6: 1090-1106, 2010.

42. Knoll G, Bittner S, Kurz M, Jantsch J and Ehrenschwender M: Hypoxia regulates TRAIL sensitivity of colorectal cancer cells through mitochondrial autophagy. Oncotarget 7: 41488-41504, 2016.

43. He W, Wang Q, Xu J, Xu X, Padilla MT, Ren G, Gou X and Lin Y: Attenuation of TNFSF10/TRAIL-induced apoptosis by an autophagic survival pathway involving TRAF2- and RIPK1/ RIP1-mediated MAPK8/JNK activation. Autophagy 8: 1811-1821, 2012.

44. Lim SC,Jeon HJ,Kee KH,Lee MJ,Hong R and Han SI: Involvement of DR4/JNK pathway-mediated autophagy in acquired TRAIL resistance in HepG2 cells. Int J Oncol 49: 1983-1990, 2016.

45. Kanzawa T, Germano IM, Komata T, Ito H, Kondo Y and Kondo S: Role of autophagy in temozolomide-induced cytotoxicity for malignant glioma cells. Cell Death Differ 11: 448-457, 2004.

46. Knizhnik AV, Roos WP, Nikolova T, Quiros S, Tomaszowski KH, Christmann M and Kaina B: Survival and death strategies in glioma cells: Autophagy, senescence and apoptosis triggered by a single type of temozolomide-induced DNA damage. PLoS One 8: e55665, 2013.

47. Guo W, Wang Y, Wang Z, Wang YP and Zheng H: Inhibiting autophagy increases epirubicin's cytotoxicity in breast cancer cells. Cancer Sci 107: 1610-1621, 2016.

48. Prieto-Domínguez N, Ordóñez R, Fernández A, García-Palomo A, Muntané J, González-Gallego J and Mauriz JL: Modulation of autophagy by sorafenib: Effects on treatment response. Front Pharmacol 7: 151, 2016.

49. Lockshin RA and Zakeri Z: Apoptosis, autophagy, and more. Int J Biochem Cell Biol 36: 2405-2419, 2004

50. Eisenberg-Lerner A, Bialik S, Simon HU and Kimchi A: Life and death partners: Apoptosis, autophagy and the cross-talk between them. Cell Death Differ 16: 966-975, 2009.

51. Mukhopadhyay S, Panda PK, Sinha N, Das DN and Bhutia SK: Autophagy and apoptosis: Where do they meet? Apoptosis 19: 555-566, 2014

This work is licensed under a Creative Commons Attribution-NonCommercial-NoDerivatives 4.0 International (CC BY-NC-ND 4.0) License. 